\title{
The impact of an arts-based programme on the affective and cognitive components of empathic development
}

\author{
Joyce Zazulak, ${ }^{1}$ Camilla Halgren, ${ }^{2}$ Megan Tan, ${ }^{2}$ Lawrence E M Grierson ${ }^{1,2,3}$
}

1 Department of Family Medicine, McMaster University, Hamilton, Ontario, Canada ${ }^{2}$ Michael G DeGroote School of Medicine, Faculty of Health Sciences, McMaster University, Hamilton, Ontario, Canada ${ }^{3}$ Program for Educational Research and Development, Faculty of Health Sciences, McMaster University, Hamilton, Ontario, Canada

\section{Correspondence to}

Dr Lawrence Grierson, Department of Family

Medicine, McMaster University, McMaster Innovation Park,

175 Longwood Rd. S., Suite 201A, Hamilton, Ontario, Canada L8P 0A1;

lawrencegrierson@gmail.com

Accepted 5 January 2015 Published Online First 5 February 2015

\section{CrossMark}

To cite: Zazulak J Halgren C, Tan M, et al. Med Humanit 2015;41: 69-74.

\section{ABSTRACT}

Medical education research demonstrates that empathic behaviour is amenable to positive change when targeted through educational programmes. This study evaluates the impact of an arts-based intervention designed to nurture learner empathy through the provision of facilitated visual literacy activities. Health Sciences students ( $N=19)$ were assigned to two learning groups: a group that participated in a visual literacy programme at the McMaster Museum of Art and a control group that participated in the normal Health Sciences curriculum. All participants completed an inter-reactivity index, which measures empathy on affective and cognitive levels, prior to and following the programme. Those individuals assigned to the visual literacy programme also completed open-ended questions concerning the programme's impact on their empathic development. The index scores were subjected to independent within-group, between-test analyses. There was no significant impact of the programme on the participants' overall empathic response. However, subcomponent analyses revealed that the programme had a significant positive effect on cognitive aspects of empathy. This finding was substantiated by the narrative reports. The study concludes that the affective focus of humanities-based education needs to be enhanced and recommends that learners are educated on the different components that comprise the overall empathic response.

\section{INTRODUCTION}

Empathy refers to one's ability to experience vicariously the feelings, thoughts, viewpoints and values of another. It is a complex construct underpinned by the interaction of cognition and affect. The cognitive aspect of empathy reflects one's ability to identify and understand the experience of others via his/her intellect. This is sometimes referred to as 'perspective-taking', and may be open and responsive to nurturing. The affective aspect refers to the passive response of an individual to the emotions of another person and, as such, are often considered a personal trait, which may be less amenable to teaching and learning. ${ }^{12}$ In the case of the physician, empathy is a critical determinant of the overall quality of care that she/he provides to her/his patients. The physician who is able to understand the perspective of another is afforded an enriched framework for understanding a patient, which can contribute to accurate decisions, ${ }^{3}$ reduce patient anxiety ${ }^{4}$ and encourage adherence. $^{5}$ Yet, a number of recent research studies have shown that medical trainees' empathy declines during training, ${ }^{2}{ }^{6-10}$ reaching its lowest levels during residency. ${ }^{1}{ }^{8} 9$ It is hypothesised that this decline is a result of cynicism that develops due to a hidden focus on detachment and clinical neutrality during medical education. ${ }^{7}$

Accordingly, the medical education community has responded by introducing a number of educational interventions designed to offset this discouraging trend and foster the development of empathic ability. ${ }^{79}$ There are communication skills workshops, which use lecture, video and audio recordings to highlight the behavioural manifestations of empathy ${ }^{7}$; experiential learning activities in which students assume the role of the patient in simulated clinical scenarios ${ }^{7}$; narrative interventions, which use literary and theatrical media to immerse trainees within the contexts of illness and care $^{11}{ }^{12}$; and new approaches that engage students in the facilitated observation of art. ${ }^{13-17}$ In each case, the implication is that the learning experience modifies the way learners process cognitive and/or affective information in a way that fosters the development of empathy.

Art appreciation techniques, which include guided observation and facilitated discussion, were first introduced to the medical education community by Dolev et $a l^{18}$ in a training programme designed to teach students how to reason physiology and pathophysiology from careful observation-an attribute they referred to as visual literacy. In Dolev's programme, and in several similar programmes, ${ }^{15} 17 \quad 19$ student participants visit the museum, observe paintings and discuss their perceptions in a facilitated group session. This educational approach is based on the results of qualitative, stream-of-consciousness interview research that describes how individuals' observational ability changes with experience along a continuum ranging from simple observer to re-creative reflector. ${ }^{20}$ The basic idea is that exposure to art appreciation techniques equips learners with visual literacy strategies that increase the attention that is paid to the details that constitute an image, which in turn improves the quality of the perceptions that are formed through observation. In this regard, visual literacy exercises purport to have a positive impact on learners' cognitive processing. Thus, in the context of patient interactions, this improved cognitive ability to recognise and process visual details may also ultimately improve a learner's ability to empathise. ${ }^{16} 17$

Along with colleagues from the Department of Family Medicine and throughout the Michael G DeGroote School of Medicine at McMaster 
University (Hamilton, Ontario), we have started to evaluate the actual effectiveness of a similar arts-based programme in improving the empathic ability displayed by medical trainees. Our educational programme, The Art of Seeing, was first piloted to a group of eight Family Medicine residents in $83 \mathrm{~h}$ sessions that were co-led by an art educator and family physician over the course of 4 months. The programme taught residents the basics of formal art analysis, challenged them to apply what they had learned to the interpretation of complex works and engaged them in narrative writing in which they reflected on the application of the programme's lessons to clinical practice. Preliminary focus group and participant surveying conducted at the end of the pilot suggested that understanding the programme's impact on empathy was worth further pursuit (see Zazulak et $a l^{17}$ for a review of The Art of Seeing).

Accordingly, the educational intervention and evaluation described in this manuscript aims to further elucidate the impact that The Art of Seeing has on the development of student empathy. This second iteration of the programme was delivered to a group of undergraduate health sciences students and included the rigorous implementation of a psychometric tool designed to assess empathy-the Interpersonal Reactivity Index (IRI) $^{21-23}$ The IRI separates the construct of empathy into a cognitive component and an affective component, each of which is underpinned by two sub-components. Specifically, the cognitive component is divided into perspective taking (PT), which describes the individual's capability to adopt the perspectives of others, and fantasy (FS), which describes the extent to which individuals can identify with characters in fictional narrative situations. The affective component, by contrast, is divided into empathic concern (EC), the compassion an individual feels for others, and personal distress (PD), which refers to feelings of anxiety and discomfort an individual experiences when observing another's negative experience. The idea is that these components and sub-components can be independent and/or interdependent with respect to their contributions to global exhibitions of empathic behaviour. To elaborate, through systematic preprogramme and postprogramme administration of the IRI to independent participatory and control groups, this evaluation hoped to capture potential changes in participant empathy associated with the programme and whether the programme had specific impacts on either the cognitive and/or emotional components of the construct. In addition, we also evaluated the way the programme impacted participants' empathy through review of answers to open-ended questions that were submitted by the participants at the end of the programme. The concept of visual literacy development suggests a potential cognitive impact; however, we remained open to the idea that a change in one's observational approach may also elicit an emotional empathic response.

\section{METHODS}

\section{Participants}

Nineteen undergraduate students enrolled in the second, third or fourth years of the Bachelor of Health Sciences programme at McMaster University (Hamilton, Ontario) volunteered to participate in this evaluation. Ten (eight women, two men) students self-identified as volunteers for The Art of Seeing and comprised our intervention group, which participated in the programme as well as preprogramme and postprogramme assessments. After completing the first session of programme, one participant from the intervention group was unable to continue as a study participant leaving nine in the intervention group. The remaining nine students (seven women, two men) had also self-identified as volunteers for the programme but were required to withdraw their interest on account of scheduling conflicts. This group of students agreed to participate in the evaluation as a genderedmatched control group, which participated in the preprogramme and postprogramme assessments but did not receive any formal intervention. None of the participants had any formal art appreciation training and all volunteers provided informed consent according to guidelines set out by Hamilton Integrated Research Ethics Board and the Declaration of Helsinki (1964).

\section{The Art of Seeing curriculum}

The intervention group participated in $42 \mathrm{~h}$ sessions held over the course of a month at the McMaster Museum of Art (MMA). Each session was cofacilitated by an art educator from the MMA and a family physician from the Department of Family Medicine at McMaster University. During the first hour of each session, the educators provided the student participants with an introduction to the technique that would be the focus of the session. This was accomplished through a series of visual examples, presented in the form of digital presentation slides that were discussed with relation to the relevant technique. In the second hour of the session, students entered the gallery to apply the technique they had learned to the works of art in the museum. The programme began with an introduction to formal analysis of a simple object. Subsequent sessions were designed to build from skills acquired in previous sessions. The intent was to guide the participants to progress from simple looking to the development of more heightened observational skills. More specifically, the four sessions included the following:

1. Introduction to Formal Analysis: In this session, participants were introduced to, and were provided a checklist of, the elements and principles of art (ie, colour, line, emphasis and movement) that are used to analyse a piece of artwork formally. Following this introduction, participants were shown figurative and abstract pieces of art and were asked to describe the works using their new terminology. The pieces of art were selected with consideration for the intended analysis from the MMA's permanent collection. This session ended with a short narrative homework assignment in which students observed and then described a person with whom they had a recent interaction with, employing techniques similar to those used in the gallery.

2. Introduction to Interpretation: In this session, participants were introduced to evidence-based looking, which describes the ability to analyse artwork with the aforementioned descriptors in a way that elucidates the meaning of the artwork and/or the artist's intentions in creating the piece.

3. Introduction to Signs and Symbols: This session introduced participants to the use of signs and symbols in art and everyday life. Building on the techniques of the previous two sessions, participants were challenged to describe and interpret the meanings contained within various signs and symbols. A specific emphasis was placed on clinical manifestations of signs and symbols (ie, body language) and their potential meaning in a healthcare context. This session also paid particular attention to the assumptions that are often made when interpreting certain stereotypical images as well as the personal stories that can underwrite signs and symbols (ie, personal stories behind an individual's tattoo choice). This session ended with another short narrative assignment in which students were asked to apply the techniques they had learned to date to write about a day in the life of a person whom they find interesting. 
4. Introduction to Conceptual and Contemporary Art: This session focused on the notion that what one observes is not always immediately recognisable. Using contemporary artwork, this session attempted to enhance the participants' tolerance for ambiguity and challenged them to consider how their own personal experiences might bias their observations and interpretations.

\section{Dependent measures and analyses}

The IRI was ultimately chosen for its ability to assess both cognitive and emotional components of empathy in a way that allows us to investigate their independent contributions to the overall construct. The IRI contains 28 items scored using a fivepoint Likert scale spanning a continuum of responses that reflect the degree to which the respondent believes the statement contained in the item is relevant to her/him. ${ }^{21-23}$ As mentioned earlier, the IRI consists of four seven-item subscales: FS, EC, PT and PD. All IRI data were analysed using the GraphPad Prism V.5.0 statistical software for Macintosh (LaJolla, California, USA). The IRI was administered to the control and intervention groups prior to the start of The Art of Seeing programme. The IRI was then readministered to both groups following the completion of the fourth session of the programme. The data were analysed using paired $t$ tests, conducted between the pretest and post-test scores for the control and intervention groups on the total IRI scores as well as on scores accrued for each of the four seven-item sub-components. A two-tailed p value of $<0.05$ was considered statistically significant.

In keeping with recent recommendations regarding the use of qualitative methods that offer additional perspectives to the understanding of empathic development, ${ }^{24}$ approximately 2 weeks after the completion of The Art of Seeing training programme and the administration of the postintervention IRI, students in the intervention group were invited to complete a short survey assignment of open-ended questions online. The questions were designed to assess whether the students felt that their empathic tendencies were enhanced as a result of participating in the programme, as well as to determine whether the programme was effective in developing skills other than visual literacy. The assignment provided the following four short answer questions as prompts for the submission:

1. Discuss whether you feel the Art of Seeing Program developed your ability to communicate/empathise with others? If possible, can you describe any examples of this?

2. Other than empathy/communication skills, please discuss what other skills you feel have been developed (if any) by The Art of Seeing programme?

3. How do you think the skills you have learned can apply to your daily life or your future endeavours?

4. Can you describe an activity that was part of the programme (museum activities and/or homework assignments) which stood out to you and what you feel it taught you?

The results of the survey assignment were evaluated by way of a template analysis, which involved organising and appraising the participants' answers to the questions prompted according to a hierarchical set of themes that were determined a priori based on the components of empathy described by the IRI tool. In this way, text that reflected upon the development of a refined empathic response was included for analysis. Given that the participant responses were prompted directly with regard to empathic development, this included most of what was written. Sections of the response text were then organised under sub-themes according to the degree that they were in reference to the cognitive or affective development of empathy. Decisions regarding this coding were facilitated by the definitions of PT and FS that constitute the cognitive contributions to the empathic response and of Empathic Concern and PD that constitute the affective contributions of the empathic response. In this way, the responses were used to substantiate or refute the results of the quantitative survey analysis. The analysis was conducted independently by three evaluators, with agreement between two evaluators necessary for each section of coded text to be included. Throughout the analysis process an additional theme regarding the duration of the programme was identified.

\section{RESULTS}

\section{Total IRI score analyses}

The analyses revealed no significant differences between the total pretest and post-test IRI scores for either the control group (pretest mean $=71.0 \pm 11.8$; post-test mean $=71.3 \pm 9.8 ; \mathrm{t}(8)$ $=0.1183, \mathrm{p}=0.91$ ) or the intervention group (pretest mean $=68.9 \pm 13.1$; post-test mean $=70.7 \pm 14.4 ; \mathrm{t}(9)=0.7311$, $\mathrm{p}=0.48$; see figure 1$)$.

\section{IRI cognition sub-component analyses}

The analyses revealed no significant PT differences between the pretest and post-test means for either the control (pretest mean $=18.9 \pm 5.2$; post-test mean $=19.6 \pm 3.1$ ) or intervention (pretest mean $=18.7 \pm 1.8$; post-test mean $=18.2 \pm 3.2$ ) groups. Similarly, no significant difference was observed between the pretest and post-test FS means for the control group (pretest mean $=16.6 \pm 5.0$; post-test mean $=17.9 \pm 3.3$ ). However, the FS analysis yielded a significant pretest versus post-test difference in the intervention group $(t(9)=2.905, p=0.0174$; figure 1 ), which described an improvement in FS at the end of the programme.

\section{IRI affective sub-component analyses}

The analyses of the EC sub-component revealed no significant differences from the comparison of pretest and post-test means

\section{Total IRI Score}

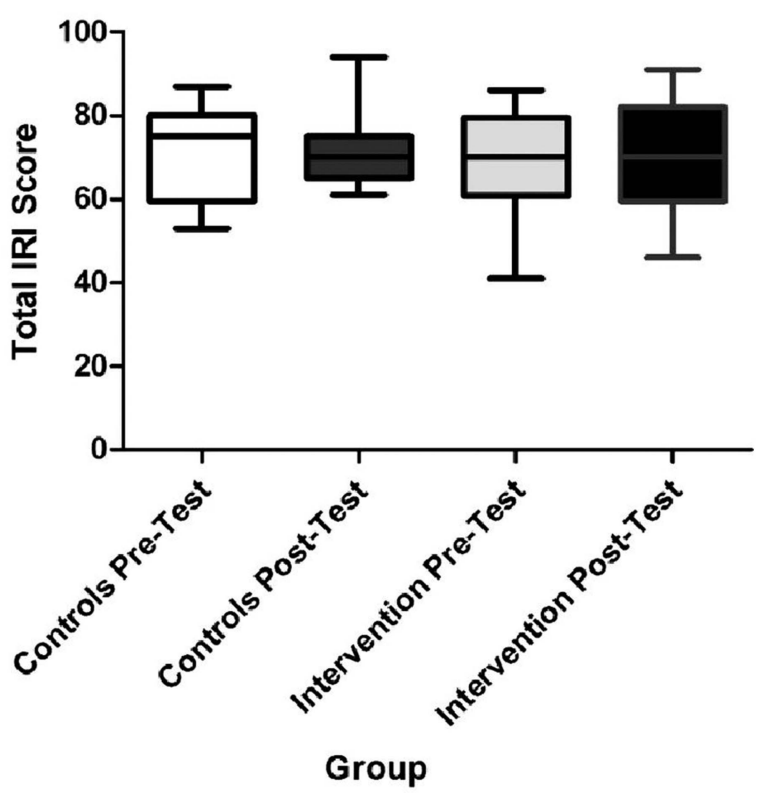

Figure 1 Total Interpersonal Reactivity Index (IRI) Score: Participants were asked to complete the IRI prior to the start of the programme and upon its completion. Paired t tests revealed no significant differences between the total pretest and post-test IRI scores for either the control or the intervention groups. 
for either the control (Pretest mean $=21.0 \pm 3.9$; Post-test mean $=21.8 \pm 3.2$ ) or intervention (Pretest mean $=20.5 \pm 3.6$; Post-test mean $=20.3 \pm 2.7$ ) groups. However, the analysis of the control group's PD pretest and post-test scores yielded a significant effect $(\mathrm{t}(8)=2.630, \mathrm{p}=0.0302$; figure 2$)$, in which PD scores decreased over the course of the programme. No significant PD differences were observed between the pretest and post-test means of the intervention group (Pretest mean $=13.6$ \pm 5.9 ; Post-test mean $=12.5 \pm 6.8$ ). This suggests that over the course of the intervention the control group appeared to be less affected than the intervention group by the negative experience of others.

\section{Survey assignment analysis}

Six of the nine students in the intervention group completed the online survey. When asked directly whether the Art of Seeing programme had any impact on the development of empathy, most of the students responded that it did not. One student [Participant 5] stated:

I don't think that my ability to empathize was enhanced over such a short time span...

The participants expressed that the programme did not improve empathy because its duration was too short. However, much of what the participants wrote suggested that they felt
A

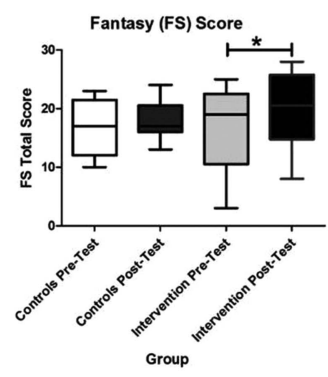

C

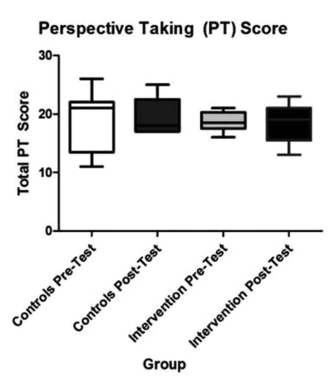

B

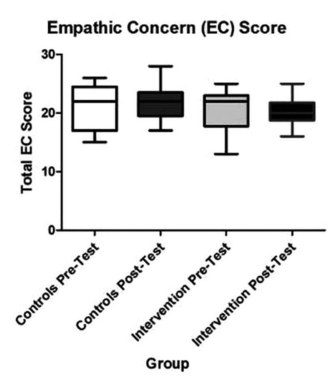

D

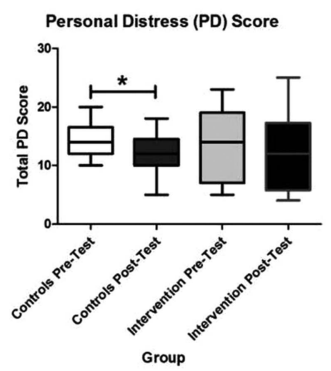

Figure 2 Interpersonal Reactivity Index (IRI) Subscale Scores: The IRI consists of four seven-item subscales. Paired t tests were conducted to analyse the pretest and post-test scores for the control and intervention groups for each subscale. All values represent mean \pm SEM. (A) Fantasy: Analyses revealed no significant difference between the pretest and post-test scores for the control group. Analyses did reveal a significant difference between the pretest and post-test scores in the intervention group $\left({ }^{*} p<0.05\right)$. (B) Empathic Concern $(E C)$ : Analyses revealed no significant differences between the pretest and post-test scores of either the control or the intervention groups. (C) Perspective Taking: Analyses revealed no significant differences between the pretest and post-test scores of either the control or the intervention groups. (D) Personal Distress: Analyses revealed a significant difference between the pretest and post-test means of the control group ( ${ }^{*} p<0.05$ ). No significant difference was observed between the pretest and post-test of the intervention group. participating in The Art of Seeing programme did indeed impact on their empathic response. For example, they reported that viewing art enabled them to become more attentive and tolerant to the feelings and viewpoints of others. One participant [P4] wrote:

I think that interpreting art is a great way of sharing different perspectives. When people look at art, they have their own interpretation and as long as they justify it, it is a logical response. The great thing is that people are forced to negotiate other people's interpretations with themselves and understand that other people's opinions are just as acceptable as their own.

\section{Another [P1] stated:}

I would say I really enjoyed the homework assignments. It allowed me an opportunity to observe and listen to other people's perspectives. Through this way, it really taught me a lot about the kind of person they are...

One student [P4] expressed this by stating:

I feel that this program helps enhance creativity, as you exercise imagination in observing a work of art. It also helps to foster tolerance for different opinions.

Furthermore, the majority of participants commented on the programme's utility in enhancing their observational skills-that is, their abilities to pick up on details that they may have previously missed. One participant [P2] responded:

\section{This program has allowed me to improve my observational skills and reflective practices... I have noticed that I am able to pick up on more details and concepts that I likely would have over- looked, had I not practiced observations and analysis in real-life situations through this program.}

Review of the participant responses also revealed a number of statements that reflect the participants' perceptions that the programme contributed to their development of abilities for greater tolerance of the viewpoints of others. This emerged most often in the participants' writing about the survey assignments. They stated that the experience of writing led to critical reflection and also noted the added benefit that they received from sharing their works with each other. One individual [P3] commented on how writing assignments and subsequent debriefs allowed him/her to understand the types of assumptions he/she makes when meeting individuals for the first time, and where these assumptions may have stemmed from:

There was a lot of space for reflection and learning things about self. For example we learned more about ourselves through the writing assignments and about what we assume/see in the people we meet

Another participant [P6] expressed a similar sentiment when speaking about the homework assignments:

[The homework assignments] gave me an opportunity to observe and listen to other people's perspectives... [It] really taught me a lot about the kind of person they are.

Taken together, each of the above cases and many of the unlisted quotes for which these stand as exemplars, the participants are, in fact, discussing a developmental impact of the Program on their PT ability-a cognitive sub-component of the empathic response.

\section{DISCUSSION}

The evaluation conducted here was designed to investigate the impact that our arts-based curriculum, known as The Art of 
Seeing, had on participants' self-reported development of empathic behaviour. The programme proposes that education in the techniques of art appreciation helps participants develop skills in visual information processing, which may be beneficial to observation that occurs outside of the museum such as during the interpersonal interactions that underpin much of clinical care. Through the use of the multidimensional IRI, we sought to test the hypothesis that this type of programme has an overall positive impact on self-reported empathy as well as the hypothesis that this type of educational intervention may have a more specific impact on one or more of the sub-components of the global construct. In particular, we reasoned that the focus of The Art of Seeing on visual literacy development would lead to a more pronounced effect on PT and FS, the cognitive subcomponents of empathy.

Although our overall results do not verify the idea of a global improvement in self-reported empathy, the findings do lend support to the second hypothesis, that is, the participants of this study's intervention group exhibited higher FS scores, a subcomponent of the cognitive aspect of empathy, on the IRI following their involvement in The Art of Seeing. This finding suggests that the programme is effective in developing one's ability to identify strongly with fictitious characters and seems reasonable when the specific activities undertaken in The Art of Seeing sessions are considered. For example, many of the art appreciation exercises challenged participants to develop narratives for the persons depicted in paintings, such as George Reid's 'A Call to Dinner', which depicts a peasant woman looking to the horizon. In this exercise, students were asked to describe the painting in detail and, subsequently, imagine a story for the woman who detailed her origins, motivations and lifestyle. Similar activities occurred throughout the four sessions and also appeared in the short homework assignments, which prompted students to develop narratives for individuals whom they had observed in their everyday lives.

The effectiveness of the programme's activities in developing the cognitive components of empathic behaviour was also supported by the responses from the survey assignment. Many of the students expressed that the programme encouraged them to appreciate the viewpoints of others. These statements highlight the participants' beliefs that the learning imparted by the programme helped them to connect with the personal viewpoints and narratives of others. Ostensibly, the participants felt that the programme developed their skills in the area of PT. Importantly, although the participants acknowledge these impacts, they state uniformly that the programme was not effective in influence their overall empathic behaviour.

Taken all together, these results present an interesting situation. In particular, when we consider the participants' opinions about experiencing no change in overall empathic behaviour, the IRI findings concerning improved FS score and their statements about enhanced PT ability, it appears that they may consider empathy as a construct that is largely affective and separate from any cognitive contributions. This means that students may understand empathy differently from how it is defined in the health professions education literature. Given the student propensity to use stated learning objectives to guide educational effort, the results presented here suggest that there may be a need to properly align students' understanding of the scope and types of actions that constitute empathic behaviour when implementing curricula designed to develop empathy.

Also noteworthy in the IRI results was a significant control group decrease in the empathy sub-component of PD-an affective construct that refers to the discomfort or anxiety that one feels when observing another's negative experience-over the course of the programme. Thus, the data suggest that The Art of Seeing may offset some early decline in empathy that is present among the participants' classmates. The potential implications of this finding are twofold: first, if this is indeed the case, it suggests that the response to the decline in trainee empathy should not focus solely on making changes to postgraduate medical education curricula. Rather, the medical education community may want to consider the ways in which a student candidate's empathic trajectory can be assessed at and/or shortly after the time of admission. Second, the absence of this decline in the intervention group suggests that the programme may have played some role in mitigating any potential decline in PD. This is important because it suggests that medical education research may need to pay more attention to aspects of cognition when seeking theory-based explanations for the decline in medical trainee empathy, which to date have largely focused on affective constructs such as distress and burnout (see Neumann et $a l^{10}$ for a review of theories of empathic decline in health professional trainees). Although, we must be cognisant of the limitations associated with making generalisations based on a small number of participants, and on findings elicited via selfreport as is the case with both the IRI tool and the survey assignment. ${ }^{25}$ However, health professional feeder programmes — such as McMaster University's Bachelor of Health Sciences programme-may consider how similar arts-based programmes designed to promote empathic development could be a worthwhile endeavour with respect to preparing their graduates for future professional success.

In conclusion, the evaluation of The Art of Seeing demonstrates the potential benefit associated with arts-based programmes in enhancing the empathic development of participants. This work extends previous efforts by showing that this type of intervention may have relevance and impact to students at a prehealth professional training stage of education and provides a strong rationale for recommendations to improve the specific impacts of facilitated art observation on empathy development. In particular, the data suggest that these types of programmes need to be cognisant of relationships and limits that exist between certain observational techniques and the individual sub-components of empathy. For instance, the current programme could potentially be improved through increased focus on art appreciation exercises that are geared towards affective aspects of empathy. Attempts to influence participants' empathic concern-an affective sub-component-may be fruitless if the selected art appreciation techniques do not develop the type of emotional sensitivity that is at the foundation of interpersonal compassion. Of course, we maintain that the implementation of any curricular changes, that are aligned with the empathy subcomponents or otherwise, should be subjected to regular and rigorous assessment that aims to capture the breadth of impact that observational education has on empathy development. These evaluations should seek to assess larger intervention groups across lengthened programmes and to incorporate a variety of methods, including survey, narrative writing, simulated and/or actual in situ assessments and structured interviews. Establishing the potential of an arts-based programme that nurtures empathy development will provide strong support for embedding arts and humanities training as important and credible parts of core curriculum in medical education.

Acknowledgements We thank Nicole Knibb and the McMaster Museum of Art for their contributions to this project. Without their support, this work would not be possible. 
Contributors JZ led the study, facilitated data collection, and wrote the manuscript. CH and MT assisted in study design, data collection and manuscript generation. LEMG supervised and contributed to all aspects of the project. All authors contributed to the critical revision of the paper, approved the final manuscript for publication, and have agreed to be accountable for all aspects of the work in ensuring that questions related to the accuracy or integrity of any part of the work were appropriately investigated and resolved.

Competing interests None.

Ethics approval Hamiliton Intergrated Research Ethics Board.

Provenance and peer review Not commissioned; externally peer reviewed.

\section{REFERENCES}

1 Davis MH. Measuring individual differences in empathy: evidence for a multidimensional approach. J Pers Soc Psychol 1983;44:113.

2 Batt-Rawden SA, Chisolm MS, Anton B, et al. Teaching empathy to medical students: an updated, systematic review. Acad Med 2013;88:1171-7.

3 Gianakos D. Empathy revisited. Arch Intern Med 1996;156:135-6.

$4 \mathrm{Kim}$ SS, Kaplowitz S, Johnston MV. The effects of physician empathy on patient satisfaction and compliance. Eval Health Prof 2004;27:237-51.

5 Bellet PS, Maloney MJ. The importance of empathy as an interviewing skill in medicine. JAMA 1991;266:1831-2.

6 Hojat M, Mangione S, Nasca TJ, et al. An empirical study of decline in empathy in medical school. Med Educ 2004;38:934-41.

7 Hojat M, Vergare MJ, Maxwell K, et al. The devil is in the third year: a longitudinal study of erosion of empathy in medical school. Acad Med 2009;84:1182-91.

8 Chen D, Lew R, Hershman W, et al. A cross-sectional measurement of medical student empathy. J Gen Intern Med 2007;22:1434-8.

9 Stepien KA, Baernstein A. Educating for empathy. J Gen Intern Med 2006;21:524-30.

10 Neumann M, Edelhäuser F, Tauschel D, et al. Empathy decline and its reasons: a systematic review of studies with medical students and residents. Acad Med 2011;86:996-1009.
11 DasGupta S, Charon R. Personal illness narratives: using reflective writing to teach empathy. Acad Med 2004;79:351-6.

12 Lie D. Point-of-view writing: a method for increasing medical students' empathy, identification and expression of emotion, and insight. Educ Health 2006;19:96-105.

13 Elder NC, Tobias B, Lucero-Criswell A, et al. The art of observation: impact of a family medicine and art museum partnership on student education. Fam Med 2006;38:393.

14 Klugman CM, Peel J, Beckmann-Mendez D. Art rounds: teaching interprofessional students visual thinking strategies at one school. Acad Med 2011;86:1266-71.

15 Naghshineh S, Hafler JP, Miller AR, et al. Formal art observation training improves medical students' visual diagnostic skills. J Gen Intern Med 2008;23:991-7.

16 Shapiro J, Rucker L, Beck J. Training the clinical eye and mind: using the arts to develop medical students' observational and pattern recognition skills. Med Educ 2006;40:263-8

17 Zazulak J, Booth K, Price D, et al. The creative art of medical inquiry. Museums Soc Issues 2010;5:250-7.

18 Dolev JC, Krohner Friedlaender L, Braverman IM. Use of fine art to enhance visual diagnostic skills. JAMA 2001;286:1020-1.

19 Kirklin D, Duncan J, McBride S, et al. A cluster design controlled trial of arts-based observational skills training in primary care. Med Educ 2007;41:395-401.

20 Housen A. Voices of viewers: iterative research, theory and practice. Arts Learn Res J 2001;17:2-12.

21 Davis MH. A multidimensional approach to individual differences in empathy. JSAS Catalog Selected Documents Psychol 1980;10:85.

22 Spinella M. Prefrontal substrates of empathy: psychometric evidence in a community sample. Biol Psychol 2005;70:175-81.

23 Sands SA, Stanley P, Charon R. Pediatric narrative oncology: interprofessional training to promote empathy, build teams, and prevent burnout. J Support Oncol 2008;6:307-12.

24 Pedersen R. Empirical research on empathy in medicine-A critical review. Patient Educ Couns 2009;76:307-22.

25 Hemmerdinger JM, Stoddart SD, Lilford RJ. A systematic review of tests of empathy in medicine. BMC Med Educ 2007;7:24-31. 